\title{
Simultaneous Radar and Video Observations of the Sea Surface in Field Conditions
}

\author{
Yurovsky Yu. Yu. ${ }^{1}$, Kudryavtsev V. N. ${ }^{1,2}$, Chapron Bertrand ${ }^{3}$
}

${ }^{1}$ RAS, FSBSI Marine Hydrophys Inst, Moscow, Russia.

${ }^{2}$ Russian State Hydrometeorol Univ, St Petersburg, Russia.

${ }^{3}$ Inst Francais Rech Exploitat Mer, Paris, France.

\begin{abstract}
:
Results of joint radar and video observations from the Black Sea Research platform are presented. Kaband dual-copolarized (VV and $\mathrm{HH}$ ) radar was used for measurements of backscattering cross-section and Doppler shifts. The radar footprint was observed by video camera, which was strictly synchronized with the radar. The total coverage of the radar footprint by bright features are computed using measured radar angular antenna pattern. Large foaming breaking waves resolved by the radar footprint are analyzed individually. Active phase of breaking wave process is accompanied by short intensive spike in radar backscattering. During the spike, the polarization ratio $(\mathrm{HH} / \mathrm{VV})$ approaches 1 , indicating specular mechanism in backscattering from disturbed water mass in breaking crest. However, consequent passage of foam through the radar footprint leads to depression in radar signal, which lasts few times longer than the spike and may reduce an overall contribution of large foaming events to the mean crosssection. In contrast to the cross-section, wave breaking events do not produce intensive spikes in Doppler velocity, suggesting that speed of the scatterer associated with breaker is less than phase speed of breaking wave. Micro-scale breakers do not generate foam, but produce short-living solar glints which are associated with anomalous slope about 40-50 degrees. Comparison of the glint coverage with instantaneous radar cross-section reveals surprisingly high correlation for both VV and $\mathrm{HH}$ polarization, suggesting that micro-scale breaking events are important in Ka-band radar backscattering. Combination of long wave properties retrieved from the Doppler velocity with video observation of the breakers allows to estimate modulation transfer function (MTF) of the wave breaking. The magnitude of such MTF decreases with wind speed and increases with wave ageing. The maximum of the large-scale wave breaking is located on the long wave crest, while the peak of microscale breaking is shifted on the long wave front face.
\end{abstract}




\section{INTRODUCTION}

Breaking of the surface waves is known to affect the signal of microwave active sensors. Backscattering from the wave breaking is usually considered $[1,2,5]$ to explain effects that cannot be predicted by classical two-scale model $[3,4]$, e.g. the ratio of co-polarized cross-sections $(\mathrm{VV} / \mathrm{HH})$, polarization ratio $(\mathrm{PR})$, which is overestimated by Bragg model. The term associated with wave breaking radar return has been phenomenologically included in the radar model [5] allowing to fit the model prediction to the real observations, but physical details of the backscattering mechanisms, as well as wave breaking itself, remain unclear.

The features of backscattering from wave breaking were investigated in many of studies $[6,7$, 8, 9]. Generally, it is supposed that steep front face of the wave prior to the breaking invokes strong and rapid specular reflection attended by short non-polarized spike in radar signal. After that backscattering from the breaker roughness supports enhancement of the cross-section level. At small grazing angles multi-bounce propagation may cause anomalous polarization ratio $(\mathrm{HH}>\mathrm{VV})$ due to the Brewster effect [10].

In [11] the X-band radar cross-section at VV polarization was compared to the coverage of a radar footprint by intensive shallow water breaking. We performed similar field experiments, but for the deep water breaking from the stationary sea-based platform. The Ka-band radar measurements were accompanied by video observation to compare the radar signal with wave breaking occurrence. Analysis of both cross-section and Doppler velocities at VV and HH polarization is performed. In this message we report the overview of the method and present the results obtained from the preliminary data examination. 


\section{EXPERIMENT}

The measurements were carried out from the Black Sea Research stationary platform. The platform is located $600 \mathrm{~m}$ offshore at $30 \mathrm{~m}$ water depth. The wave fetch varies from one to few hundred kilometers depending on wind direction, but most of the data are collected at maximal fetch (east wind) under conditions similar to the open sea. The median wave age, $c_{\mathrm{p}} / U$, is 0.75 , where $U$ is wind speed varying within $0-20 \mathrm{~m} / \mathrm{s}$, and $c_{\mathrm{p}}$ is phase speed of waves corresponding to the spectral peak, which frequency is typically higher than $0.2 \mathrm{~Hz}$. Thus shallow water effects can be disregarded.

The Ka-band continuous wave dual co-polarized ( $\mathrm{VV}$ and $\mathrm{HH}$ ) scatterometer was used for the radar measurements (see [12] for detailed description of this instrument). The radar was equipped with digital camera (1080p, FullHD) that was fixed on the radar body and co-aligned with its principal axis. Calibration of the entire system was done by observation of the spherical target, placed on the free space background at various viewing angles. The received power was then mapped over the video frame (instantaneous target positions) yielding two-way angular antenna pattern, $\Gamma_{\mathrm{pp}}$, where $\mathrm{pp}$ stands for polarization.

Synchronization between the camera and the radar was made by recording of camera audio channel by the same ADC unit that was used for the radar signal acquisition. A sophisticated correlation analysis was then performed to match the audio tracks from video and radar sources. The accuracy of the synchronization is of order of inverse sound sampling rate $(44 \mathrm{kHz})$ that is much smaller than video sequence time resolution $(25 \mathrm{~Hz})$.

The radar with camera were installed on the top (13 m height) or bottom ( $6.5 \mathrm{~m}$ height) deck of the platform depending on desirable incidence angle, which was varied from 0 to $70^{\circ}$. A supplementary meteorological data were collected by commercial weather station and custom six-wire wave gauge.

Raw radar data processing consisted in the estimation of quasi-instantaneous signal variance (0th moment of Doppler spectrum) and Doppler frequency shifts (1st moment of Doppler spectrum) over $0.2 \mathrm{sec}$ intervals that were then recalculated into quasi-instantaneous normalized radar cross-section (NRCS) and Doppler velocity (DV).

Raw video images were detrended using mean image intensity over 1 min interval. Bright features were extracted by binarizing the images with the threshold determined from the brightness distribution by the method presented in [13]. Fraction of the radar footprint area covered by wave breaking, $Q$, was estimated using measured radar patterns:

$$
Q_{\mathrm{pp}}=\frac{\int \Gamma_{\mathrm{pp}}(x, y) I(x, y) \mathrm{d} x \mathrm{~d} y}{\int \Gamma_{\mathrm{pp}}(x, y) \mathrm{d} x \mathrm{~d} y}
$$

where $\Gamma_{\mathrm{pp}}(x, y)$ is the radar pattern projected on the sea surface plane, $I(x, y)$ is the binarized image projected on the sea surface.

Note, wave breaking coverages determined in this way are slightly different for $\mathrm{VV}$ and $\mathrm{HH}$ polarizations, because of difference of $\mathrm{VV}$ and $\mathrm{HH}$ patterns. The validity of the estimated coverages is checked by comparison with empirical wind dependencies for $Q$ [14].

The radar Doppler channel is used as a sensor for long waves (LW) resolved by the antenna footprint [15]. Assuming that the DV is a measure of LW orbital velocity, the relation between DV and LW elevation is:

$$
\tilde{v}=\tilde{a} \omega(\cos \phi \sin \theta+i \cos \theta)
$$

where $\tilde{v}$ is the complex amplitude of DV, $\tilde{a}$ is the LW complex amplitude, $\theta$ is the incidence angle, $\phi$ is the radar-to-wave azimuth, $\omega$ is the LW angular frequency.

\section{RESULTS}

To demonstrate main features that can be deduced from the synchronized radar-video measurements we focus on the two particular records taken at large incidence angles $\left(53^{\circ}\right.$ and $\left.48^{\circ}\right)$ for which breaking wave influence is expected to be strong.

\subsection{Developing sea. Large-scale breakers}

The first case corresponds to the pure wind sea without swell with rather young wave age, $c_{\mathrm{p}} / U=$ 0.56 , wind speed $U=11 \mathrm{~m} / \mathrm{s}$, wave peak frequency $f_{\mathrm{p}}=0.24 \mathrm{~Hz}$ and significant wave height $H_{\mathrm{s}}=0.8 \mathrm{~m}$. The measurements were done at incidence angle $\theta=53^{\circ}$ and radar-to-wind azimuth $\phi=30^{\circ}$ (a 5 min piece of the record is shown in the Fig. 1). Under these conditions the group 


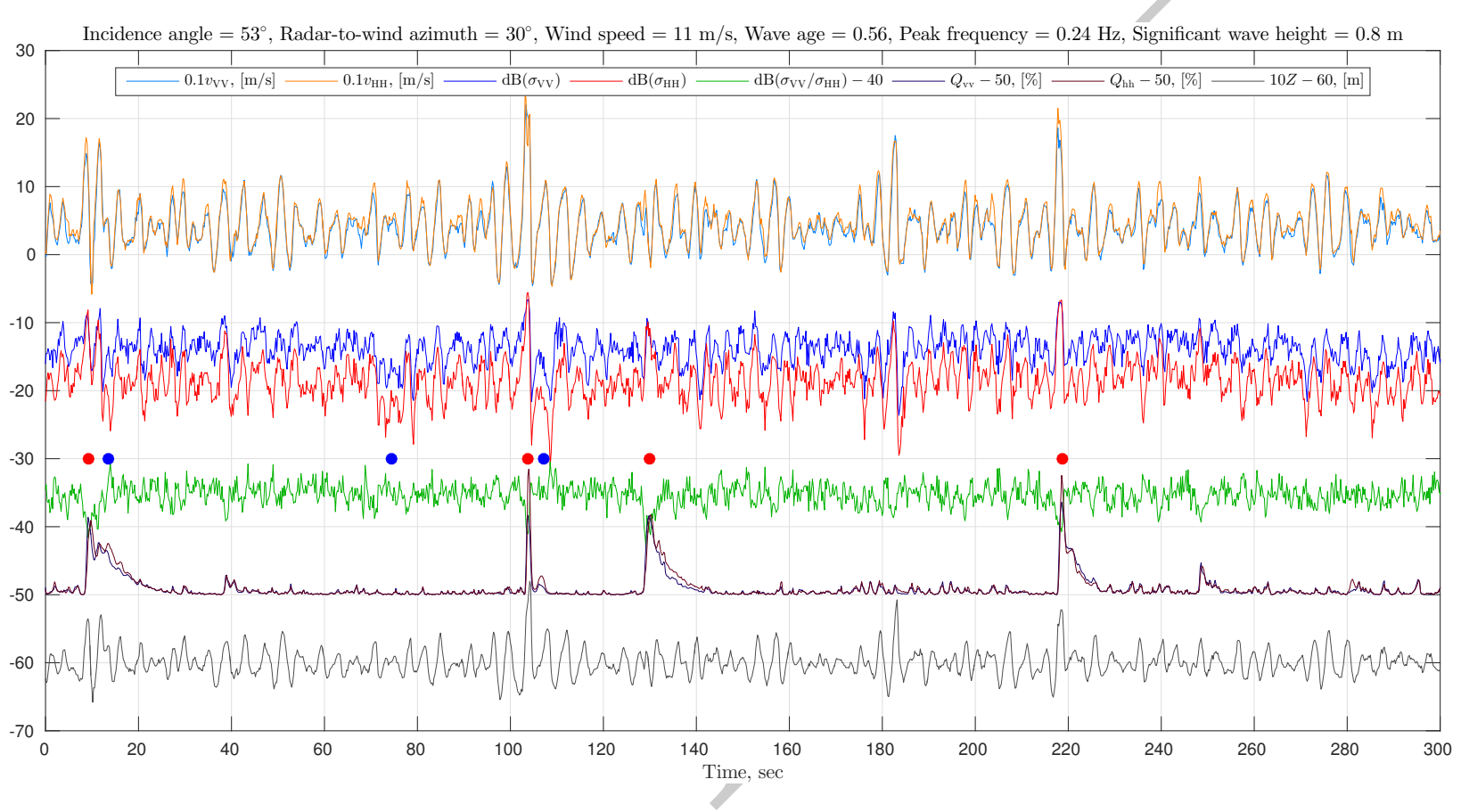

Figure 1: Pure wind sea. Time series of Doppler velocity ( $v$, sky blue - VV, orange $-\mathrm{HH})$, normalized radar cross-section $(\sigma$, blue $-\mathrm{VV}$, red $-\mathrm{HH})$, polarization ratio $\left(\sigma_{\mathrm{vv}} / \sigma_{\mathrm{hh}}\right.$, green $)$, wave breaking coverage $(Q$, dark blue - VV, dark red - HH), and surface elevation reconstructed from Doppler signal ( $Z$, gray). The signals are artificially biased for better visualization. Red symbols indicate spikes, blue symbols - pauses.

structure of the waves is evident and large wave breaking events are normally related to the elevation envelope maxima. Breaking surface during the events covers up to $10 \%$ of the effective footprint area. It is enough to produce NRCS spikes, which exceed regular peaks, related to the non-breaking wave crests, by upto $5-7 \mathrm{~dB}$.

The active and passive phases [14] can be clearly seen in the $Q$ time series: short spike (active phase) is followed by exponential decay (foam). Noticeably, the radar responds positively only to the active phase. These NRCS spikes are accompanied by negative spikes in the $\mathrm{PR} \approx 1$ indicating strong non-polarized return.

After the spike in the NRCS a some kind of pauses in signal intensity is sometimes evident. The pauses correspond to the sea surface flattening occurring in the wave breaking wakes that visually are very similar to the turbulent ship wakes. An interesting feature, that may be important in the radar models, is that the pauses are of the same negative magnitude as spikes (in decibels), but lasts few times longer: $7-10 \mathrm{sec}$ against $0.5-1 \mathrm{sec}$ for spikes. This may reduce an overall contribution of large foaming wave breaking events to the mean NRCS.

Noticeably, the spikes in $Q$ are not always followed by the passive foam (Fig. 1, $t=103 \mathrm{sec}$ ). The NRCS pauses are not always follows the NRCS spike $(t=74 \mathrm{sec})$, and the spikes are not always followed by pauses $(t=218 \mathrm{sec})$. Such a mutability may be just a result of spatial wave breaking randomness causing an appearance of random wave breaking stage in the radar footprint.

Another important feature is DV response to the wave breaking. The variation of the DV around its mean level is determined by orbital velocity amplitude, which is about $1 \mathrm{~m} / \mathrm{s}$. One could anticipate that wave breaking velocity $c_{\mathrm{wb}}$, and its corresponding DV spike $v_{\mathrm{wb}}=c_{\mathrm{wb}} \sin \theta$, should be of order of breaking wave phase velocity. If breaking of spectral peak wave is supposed, then its phase velocity should be $c_{\mathrm{p}}=6.4 \mathrm{~m} / \mathrm{s}$ and $v_{\mathrm{wb}}=5.1 \mathrm{~m} / \mathrm{s}$. However, the DV spikes during the spikes in $Q$ are not more than $v_{\mathrm{wb}} \approx 2 \mathrm{~m} / \mathrm{s}$, suggesting than wave breaking phase velocity is not more than $c_{\mathrm{wb}}=v_{\mathrm{wb}} / \sin \theta \approx 2.5 \mathrm{~m} / \mathrm{s}$ and its wave-length is about $\lambda \approx 2.5 \mathrm{~m}$. The corresponding breaking crest length, $\Lambda=0.1 \lambda$ [16], or scatterer linear size is around $25 \mathrm{~cm}$. This size is much less then really observed, thus the response of wave breaking in the DV is much less than wave breaking phase speed. 




Figure 2: Decaying sea. Notations are the same as for in Fig.1.

\subsection{Decaying sea. Small-scale breakers}

The second case corresponds to the swell dominated sea $\left(c_{\mathrm{p}} / U=1.54, U=5 \mathrm{~m} / \mathrm{s}, f_{\mathrm{p}}=0.19 \mathrm{~Hz}\right.$, $H_{\mathrm{s}}=0.7 \mathrm{~m}$ ). Under these conditions the large foaming wave breaking is quite rare event (Fig.2). However, some background level of $Q \approx 1 \%$ is always present. Visual examination of the video records suggest that the events contributing to $Q$ are the small-scale short-living highlights that are very similar to solar glints. But for the particular radar/camera look geometry and sun position, the surface patch should have slope about $40-50^{\circ}$ to provide specular reflection from the sun to the camera. Therefore, the events detected in this record are small-scales breakers.

The most surprising feature is that NRCS is highly sensitive to this micro-scale breaking (in contrast to Fig. 1, $Q$ is shown in decibels). While VV NRCS demonstrate some additional source of backscattering, presumably Bragg-like, the HH NRCS seem to be totally controlled by $Q$. Although the similar levels of NRCS and $Q$ is just a coincidence, the correlation between these two parameters is impressive. Noticeably, both NRCS and $Q$ has the same low frequency fluctuations.

In contrast to the developing sea, in absence of large-scale breakers the DV has no clear spikes and micro-scale breakers increase the mean DV level.

\subsection{Wave Breaking LW distribution and MTF}

Using relationship (2) the LW phases, and thus the distributions of the measured parameters over the LW profile, can be estimated. The distributions of backscattering characteristics and wave breaking coverage are compared in Fig. 3 for the two cases discussed above.

In both cases the NRCS has a peak at around $+45^{\circ}$ on the front LW face, indicating a balance between tilt and hydrodynamical modulation mechanisms. In the case of developing sea (Fig. 3a) with frequent occurrence of large-scale breaking, the maximum of $Q$ is located at the wave crest, but the distribution is significantly skewed towards rear slope. This is explained by presence passive foam that falls behind the breaking wave crest. In case of decaying sea (Fig. 3a) with more typical small-scale breakers, the peak of $Q$ is shifted $+45^{\circ}$ on the front face, and the distribution shape is quite symmetrical because of absence of foam.

Notice, for quite similar look geometry the NRCS peak has roughly the same place for both cases, and its position is the same as for micro-scale breaking, but not as for the large-scale events. From this observation, we can speculate that micro-scale source is still important for the first, developing sea case, but is not so well-pronounced because of different sun/video imaging geometry.

Interestingly, the PR, in terms of Bragg-like return only, cannot be affected by hydrodynamical modulation and should has minimum at $+90^{\circ}$ due to the tilting modulation. But it also has a 

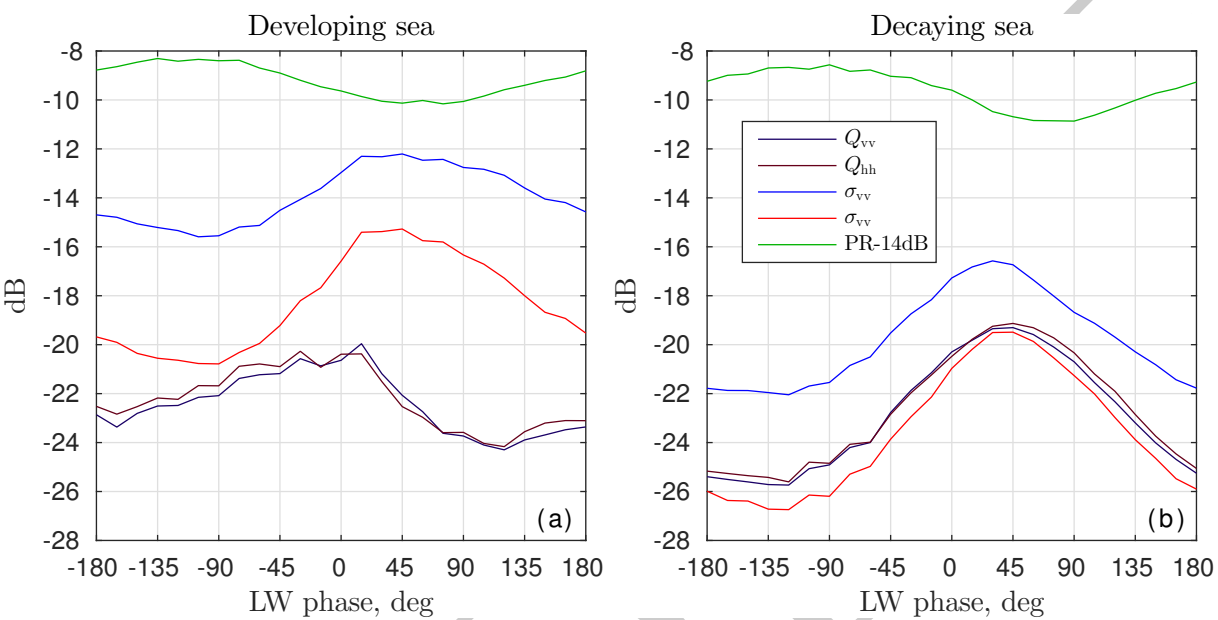

Figure 3: Distributions of NRCS, PR, and wave breaking coverage along LW phase estimated from VV DV for (a) developing sea (the same as Fig. 1) and (b) decaying sea (the same as Fig. 2). Positive phase corresponds to the front LW slope.
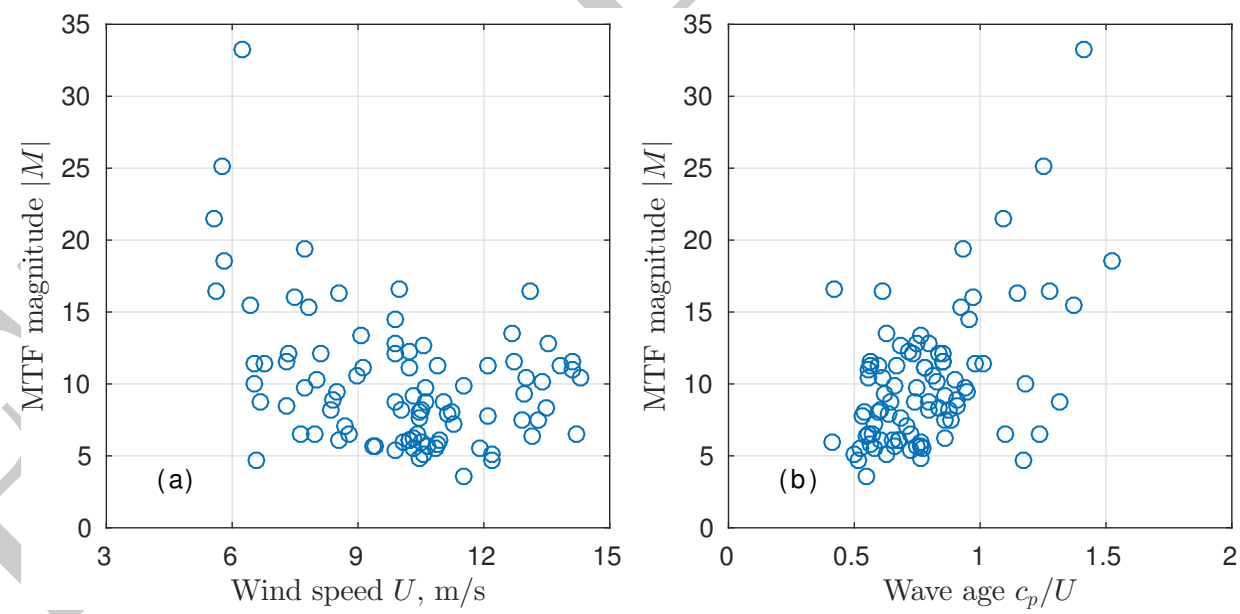

Figure 4: Magnitude of wave breaking MTF as function of (a) wind speed, $U$, and (b) wave age, $c_{\mathrm{p}} / U$.

minimum at about $+45^{\circ}$. If the micro-scale breaking really occurs at this LW phase and generates non-polarized return, then it should produce such a PR dip there.

Finally, having values of $Q$ synchronized with LW phase, extracted from the radar DV, the MTF of wave breaking coverage can be estimated:

$$
M=\frac{Q^{\prime}}{\bar{Q} a k},
$$

where $Q^{\prime}$ is the wave breaking coverage variation, $\bar{Q}$ is the mean level of $Q, k$ is the LW wavenumber.

The MTF magnitude computed for all records increases with decreasing of wind speed and increasing of wave age (Fig. 4). This is in agreement with previously reported estimates [17]. However our magnitude level is somewhat lower than their mean value $|M|=24$. This disagreement may be caused by difference in size of minimal resolved events. Resolving of small-scale events leads to increase of $\bar{Q}$, and therefore decreases estimated MTF magnitude.

\section{SUMMARY}

In this presentation we report the results of joint radar and video observations of the sea surface. Experiments were carried out from Black Sea Research platform. Ka-band dual-copolarized (VV and $\mathrm{HH}$ ) radar was used for measurements of backscattering cross-section and Doppler shifts. The 
image of radar footprint was captured by video camera, which was strictly synchronized with the radar. An auxiliary wave elevation and wind speed measurements were also done. Such a complex acquisition system allows to control how backscatter responds to various sea surface features.

Radar data are transformed into quasi-instantaneous radar cross-section and Doppler shift time series by averaging of raw radar signal over short $(0.2 \mathrm{~s})$ time intervals. Video sequences at $25 \mathrm{~Hz}$ frame rate are thresholded to extract bright features, which are active breaking waves and relict foam. The total coverage of the radar footprint by bright features are computed using measured radar angular antenna pattern.

We focus on two situations corresponding to the developing and decaying seas.

Large-scale events, typical for the developing sea, are rare and can be analyzed individually. Active phase of breaking wave process is accompanied by short intensive spike in radar backscattering cross-section. During the spike polarization ratio $(\mathrm{HH} / \mathrm{VV})$ tends to 1 , indicating specular mechanism in backscattering from disturbed water mass in the breaking crest. Following passage of foam through the radar footprint results in depression of the radar signal, which is longer than spike. Therefore this effect may reduce at extent an overall contribution of large foaming events to the NRCS. The Doppler velocity is not strongly affected by the wave breaking event, thus the velocity of the scatterer associated with breaker is lower than breaking wave phase velocity.

Micro-scale breakers, evident in the decaying sea case, do not produce foam, but transform wave energy into small-scale roughness and parasitic capillary ripples. In contrast to the foaming whitecaps, micro-scale breakers cover the footprint ubiquitously and contribute to the average signal, meaning that spikes from them are unresolved. The micro-scale breakers produce a lot of individual short-living glints having slopes about $40-50^{\circ}$ that cannot be associated with regular surface. Comparison of the footprint coverage with instantaneous radar cross-section reveals surprisingly high correlation for both VV and $\mathrm{HH}$ polarization, suggesting that micro-scale events are important in Ka-band radar backscattering.

A conjunction of radar and video camera allows to investigate wave breaking properties with the use of radar Doppler channel as a wave probe. Particularly, it is shown that large-scale events are located on the long wave crest, but residual foam moves the total (active and passive) coverage on the rear wave slope. The micro-scale breaking maximum is shifted $+45^{\circ}$ towards front slope. The magnitude of wave breaking MTF is shown to decrease wind speed and increase with wave age.

\section{ACKNOWLEDGMENT}

The work was supported by Russian Science Foundation grant 15-17-20020.

\section{REFERENCES}

1. Kalmykov, A. I. and V. V. Pustovoytenko, "On polarization features of radio signals scattered from the sea surface at small grazing angles," J. Geophys. Res. (Oceans), Vol. 81, 1960-1964, 1976.

2. Phillips, O. M. "Radar returns from the sea surface - bragg scattering and breaking waves," J. Phys. Oceanogr., Vol. 18, 1063-1074, 1988.

3. Bass, F. G., I. M. Fuks, A. I. Kalmykov, I. E. Ostrovsky, and A. D. Rosenberg, "Very high frequency radiowave scattering by a disturbed sea surface. part i: Scattering from a slightly disturbed boundary, part ii: Scattering from an actual sea surface," IEEE Trans. Ant. Propag., Vol. 16, No. 5, 554-568, 1968.

4. Wright, J. "A new model for sea clutter," IEEE Trans. Ant. Propag., Vol. 16, No. 2, 217-223, 1968.

5. Kudryavtsev, V., D. Hauser, G. Caudal, and B. Chapron, "A semiempirical model of the normalized radar cross-section of the sea surface 1. Background model," J. Geophys. Res. (Oceans), Vol. 108, No. C08054, doi: 10.1029/2001JC001003, 2003.

6. Kwoh, D. S. W. and B. M. Lake, "A deterministic, coherent, and dual-polarized laboratory study of microwave backscattering from water waves, part 1: Short gravity waves without wind," IEEE J. Ocean. Eng., Vol. 9, No. 5, 291-308, 1984.

7. Ericson, E. A., D. R. Lyzenga, and D. T. Walker, "Radar backscattering from stationary breaking waves," J. Geophys. Res. (Oceans), Vol. 104, No. C12, 29679-29695, 1999.

8. Dano, E. B., D. R. Lyzenga, and M. Perlin, "Radar backscattering from mechanically generated transient breaking waves - part 1: Angle of incidence dependence and high resolution surface morphology," IEEE J. Ocean. Eng., Vol. 26, No. 2, 181-200, 2001. 
9. Dano, E. B., D. R. Lyzenga, G. Meadows, L. Meadows, H. van Sumeren, and R. Onstott, "Radar backscattering from mechanically generated transient breaking waves - part 2: Azimuthal and grazing angle dependence," IEEE J. Ocean. Eng., Vol. 26, No. 2, 201-215, 2001.

10. West, J. C., J. M. Sturm, and S. Ja, "Low-grazing scattering from breaking water waves using an impedance boundary MM/GTD approach," IEEE Trans. Ant. Propag., Vol. 46, 93-100, 1998.

11. Haller, M. and D. Lyzenga, "Comparison of radar and video observation of shallow water breaking waves," IEEE Trans. Geosci. Rem. Sens., Vol. 41, No. 4, 832-844, 2003.

12. Yurovsky, Yu. Yu., V. N. Kudryavtsev, S. A. Grodsky, and B. Chapron, "Ka-Band Dual Copolarized Empirical Model for the Sea Surface Radar Cross Section," IEEE Trans. Geosci. Rem. Sens., doi:10.1109/TGRS.2016.2628640, 1-19, 2016.

13. Mironov, A. S. and V. A. Dulov, "Detection of wave breaking using sea surface video records," Meas. Sci. Technol., Vol. 19, No. 015405, 2008.

14. Monahan, E. C. and D. K. Woolf, "Comments on "Variations of Whitecap Coverage with Wind stress and Water Temperature," J. Phys. Oceanogr., Vol. 19, 706-709, 1989.

15. Keller, W. C. and J. W. Wright, "Microwave scattering and the straining of wind-generated waves," Radio Sci., Vol. 10, 139-147, 1975.

16. Babanin, A. V. "Breaking of ocean surface waves," Act. Phys. Slovaca, Vol. 59, 305-535, 2009.

17. Dulov, V. A., V. N. Kudryavtsev, and A. N. Bolshakov, "A Field Study of White Caps Coverage and its Modulations by Energy Containing Waves", Gas Transfer at the Water Surfaces, AGU Geophysical Monographs, Chapter 127, 187-192, 2002.

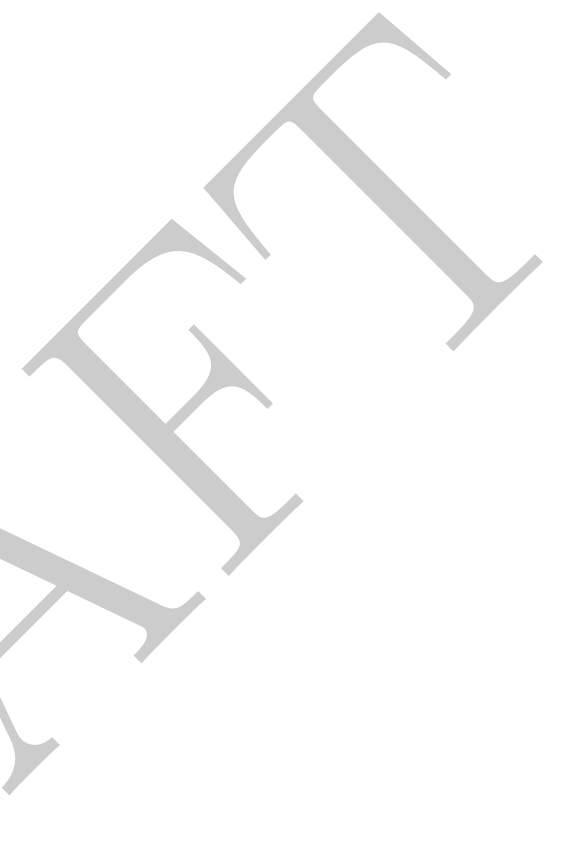

